\title{
Authors' correction
}

\section{"Efficacy and safety of budesonide/formoterol in the management of chronic obstructive pulmonary disease". W. Szafranski, A. Cukier, A. Ramirez, G. Menga, R. Sansores, S. Nahabedian, S. Peterson, H. Olsson. Eur Respir J 2003; 21: 74-81.}

Unfortunately, there was an error in the 12-month, total symptom scores data in table 4 . The correct version of table 4 is reproduced below.

Table 4. - Symptom scores for first week of treatment and at 12 months

\begin{tabular}{|c|c|c|c|c|c|c|c|c|c|c|}
\hline \multirow[t]{2}{*}{$\begin{array}{l}\text { Treatment } \\
\text { comparisons }\end{array}$} & \multicolumn{2}{|c|}{$\begin{array}{l}\text { Total symptom } \\
\text { score }(0-16)\end{array}$} & \multicolumn{2}{|c|}{$\begin{array}{l}\text { Awakening } \\
\text { score }(0-4)\end{array}$} & \multicolumn{2}{|c|}{$\begin{array}{c}\text { Shortness of } \\
\text { breath score }(0-4)\end{array}$} & \multicolumn{2}{|c|}{$\begin{array}{l}\text { Cough score } \\
(0-4)\end{array}$} & \multicolumn{2}{|c|}{$\begin{array}{l}\text { Chest tightness } \\
\text { score }(0-4)\end{array}$} \\
\hline & Week 1 & 12 months & Week 1 & 12 months & Week 1 & 12 months & Week 1 & 12 months & Week 1 & 12 months \\
\hline $\begin{array}{l}\text { Budesonide/formoterol } \\
\text { versus placebo }\end{array}$ & $\begin{array}{c}-1.21 \\
(\mathrm{p}<0.001)\end{array}$ & $\begin{array}{c}-1.12 \\
(\mathrm{p}<0.001)\end{array}$ & $\begin{array}{c}-0.30 \\
(\mathrm{p}<0.001)\end{array}$ & $\begin{array}{c}-0.34 \\
(\mathrm{p}<0.001)\end{array}$ & $\begin{array}{c}-0.35 \\
(\mathrm{p}<0.001)\end{array}$ & $\begin{array}{c}-0.36 \\
(\mathrm{p}<0.001)\end{array}$ & $\begin{array}{c}-0.28 \\
(p<0.001)\end{array}$ & $\begin{array}{c}-0.19 \\
(p=0.002)\end{array}$ & $\begin{array}{c}-0.24 \\
(\mathrm{p}<0.001)\end{array}$ & $\begin{array}{c}-0.21 \\
(\mathrm{p}<0.001)\end{array}$ \\
\hline $\begin{array}{l}\text { Budesonide/formoterol } \\
\text { versus budesonide }\end{array}$ & $\begin{array}{c}-0.80 \\
(\mathrm{p}<0.001)\end{array}$ & $\begin{array}{c}-0.84 \\
(\mathrm{p}<0.001)\end{array}$ & $\begin{array}{c}-0.20 \\
(p=0.004)\end{array}$ & $\begin{array}{c}-0.20 \\
(p=0.003)\end{array}$ & $\begin{array}{c}-0.28 \\
(\mathrm{p}<0.001)\end{array}$ & $\begin{array}{c}-0.26 \\
(\mathrm{p}<0.001)\end{array}$ & $\begin{array}{c}-0.15 \\
(\mathrm{p}=0.022)\end{array}$ & $\begin{array}{c}-0.22 \\
(\mathrm{p}<0.001)\end{array}$ & $\begin{array}{c}-0.14 \\
(\mathrm{p}=0.020)\end{array}$ & $\begin{array}{c}-0.13 \\
(\mathrm{p}=0.043)\end{array}$ \\
\hline $\begin{array}{l}\text { Budesonide/formoterol } \\
\text { versus formoterol }\end{array}$ & $\begin{array}{c}-0.69 \\
(\mathrm{p}<0.001)\end{array}$ & $\begin{array}{c}-0.41 \\
(\mathrm{p}=0.043)\end{array}$ & $\begin{array}{c}-0.20 \\
(p=0.005)\end{array}$ & $\begin{array}{c}-0.16 \\
(p=0.019)\end{array}$ & $\begin{array}{c}-0.13 \\
(\mathrm{p}=0.038)\end{array}$ & $\begin{array}{c}-0.11 \\
(\mathrm{p}=0.107)\end{array}$ & $\begin{array}{c}-0.17 \\
(\mathrm{p}=0.012)\end{array}$ & $\begin{array}{c}-0.08 \\
(p=0.204)\end{array}$ & $\begin{array}{c}-0.12 \\
(p=0.047)\end{array}$ & $\begin{array}{c}-0.03 \\
(\mathrm{p}=0.678)\end{array}$ \\
\hline
\end{tabular}

Data are presented as score (p-value). 